\title{
Intraventricular haemorrhage and stage 3 retinopathy of prematurity
}

\author{
Patrick Watts, G G W Adams, R M Thomas, Catey Bunce
}

\begin{abstract}
Backgroundlaims-A recent report has highlighted the decreasing prevalence in recent years of severe intraventricular haemorrhage (IVH) in very low birthweight (VLBW) infants $(<1500 \mathrm{~g})$. This study attempted to identify the severity of the grade of IVH in infants with stage 3 retinopathy of prematurity (ROP), and to re-examine the association between threshold ROP and IVH.
\end{abstract}

Methods-This was a retrospective study carried out over 3 years, between December 1995 and December 1998 of neonates admitted to a single neonatal intensive care unit. 28 infants with stage 3 ROP were identified from the ROP screening database. Cranial ultrasound scans were available on 24 of these infants. The scans were reviewed and the severity of IVH was graded from grade 1 to grade 4 . The birth weight, sex, ethnic origin, and gestational age of the babies were recorded. The number of infants progressing to threshold disease and the treatment provided was documented.

Results-The 24 infants had a median gestational age of 26 weeks (range 24-28 weeks) and a median birth weight of $762.5 \mathrm{~g}$ (range 540-1010 g). 17 infants were treated for threshold disease. 13 infants $(54.2 \%)$ had IVH, of these eight $(61.5 \%)$ had grade 1 , two $(15.4 \%)$ had grade 2 , one $(7.7 \%)$ had grade 3 , and two $(15.4 \%)$ had grade 4.12 of the 13 infants $(92.3 \%)$ with IVH had treatment with laser or cryotherapy for ROP compared with five of the remaining 11 infants $(p=0.023$, Fisher's exact test). These data provide little evidence of any association between IVH and each of ethnic origin $(p=0.856)$, sex $(p=1)$, birth weight, or gestational age ( $p$ $=0.56$ and $p=0.06$ respectively) in infants with stage 3 ROP.

Conclusions-These data provide strong evidence $(p=0.023)$ of an association between the presence of IVH and treatment of threshold ROP. Although the numbers in this study are small the majority of infants with stage 3 ROP had grade 1 IVH, which heralds a more favourable neurological outcome. An association between the severity of ROP and severity of IVH was not demonstrated. With improvements in neonatal care and a reduction in the prevalence of severe IVH, there appears to be a weakening of the previously reported association between severe IVH and severe ROP. However, the presence of even a minor grade of IVH may be a significant risk factor for threshold ROP once stage 3 disease is encountered.

(Brf Ophthalmol 2000;84:596-599)

The National Institute of Child Health and Human Development Neonatal Research Network register has reported an increasing survival of infants with a birth weight of 501-1500 g (VLBW). ${ }^{1}$ A large population based study of surviving VLBW infants revealed a greater risk of poor vision, myopia, and strabismus in infants with retinopathy of prematurity (ROP) compared with those without ROP. ${ }^{2}$ The incidence of intraventricular haemorrhage (IVH) in premature VLBW infants has been reported to be about $43 \%^{3}$ with previous studies reporting a significant association between the severity of IVH and severe ROP. Hungerford et al reported IVH in $79 \%$ of infants with any stage ROP and in $78 \%$ of infants with permanent ocular sequelae. ${ }^{4} \mathrm{Ng}$ et al described six infants with stage 3 ROP or greater with periventicular leucomalacia (PVL) and proposed hypoxia as a common aetiological factor. ${ }^{5}$ Recently, Cooke ${ }^{6}$ has reported that the improved survival of VLBW infants since 1990 has been accompanied by a fall in the incidence of parenchymal lesions and cerebral palsy in survivors and attributed this change to the advent of the widespread use of antenatal steroid prophylaxis.

Since the introduction of the international classification for ROP, ${ }^{7}$ the term "severe" ROP described in association with $\mathrm{IVH}^{4}{ }^{5}$ has been replaced by stage 3 ROP or worse. The aims of this study were

(1) to describe the various grades of IVH present in stage 3 ROP,

(2) to describe its association with sex, ethnic origin, birth weight, gestational age, and treatment of threshold ROP, and

(3) to re-examine the association between IVH and stage 3 ROP in view of the recent report of decreasing severity of IVH in VLBW infants. ${ }^{6}$

Patients and methods

During the period between December 1995 and December 1998, 179 neonates were admitted to a single neonatal intensive care unit in Northwick Park Hospital. The ROP screening was carried out weekly and details recorded in an ROP screening diary and in the hospital records. Screening criteria were based on the guidelines recommended by the Royal College of Ophthalmologists and the British Association of Perinatal Medicine, ${ }^{8}$ which 


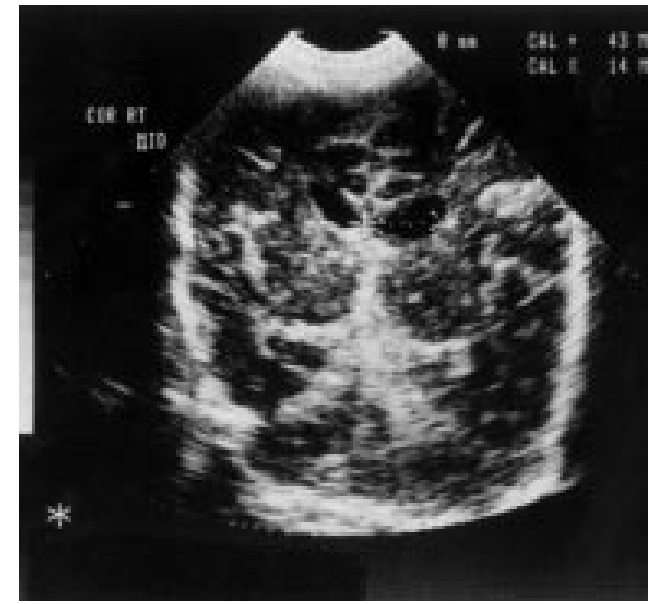

Figure 1 Coronal ultrasound scan demonstrating an intermediate grade 1/grade 2 intraventricular haemorrhage (IVH).

included babies with a birth weight of less than $1501 \mathrm{~g}$ or a gestational age of 31 weeks or less. Threshold ROP defined as stage 3 ROP in zone 1 or 2 , involving 5 contiguous clock hours or 8 cumulative clock hours in the presence of plus disease was indication for treatment and was present in $11.1 \%$ of babies ( 21 infants).

Acute ROP developed in 58 infants $(32.4 \%)$. The maximum stage of ROP reached before regression started or treatment initiated was stage 1 in 22 infants $(12.2 \%)$, stage 2 in eight infants $(4.4 \%)$, and stage 3 in 28 infants $(15.6 \%)$.

Of the 28 infants with stage 3 ROP ultrasound data were available on the 24 inborn infants, the other four babies were transferred to the unit after delivery elsewhere.

The ultrasound scans were performed on the first day of admission to the neonatal unit and several times in the first 10 days of life with a 5 or $7.5 \mathrm{MHz}$ ultrasound probe, through the anterior fontanelle, obtaining coronal, midsagittal and parasagittal right and left views. The scans were repeated at 6 weeks of age to look for signs of cystic changes. A consultant neonatologist reviewed all scans. The scans were classified in accordance with the grading of Papile $e t a l^{3}$ :

Grade $0=$ no evidence of IVH,

Grade $1=$ subependymal haemorrhage,

Grade $2=$ IVH without ventricular dilatation (Fig 1)

Grade $3=$ IVH with ventricular dilatation (Fig 2)

Grade $4=$ IVH, parenchymal haemorrhage (PH) (Fig 3).

The birth weight, gestational age, sex, ethnic origin, and any treatment for ROP were recorded. The association between IVH (grade 1 or above versus grade 0 ) and birth weight and gestational age was assessed using the Wilcoxon rank sum test. Fisher's exact test was used to assess the association between IVH and each of ethnic origin, sex, and threshold disease.

\section{Results}

Of the 179 neonates screened 121 had no ROP, 22 had stage 1, eight had stage 2 , and 28

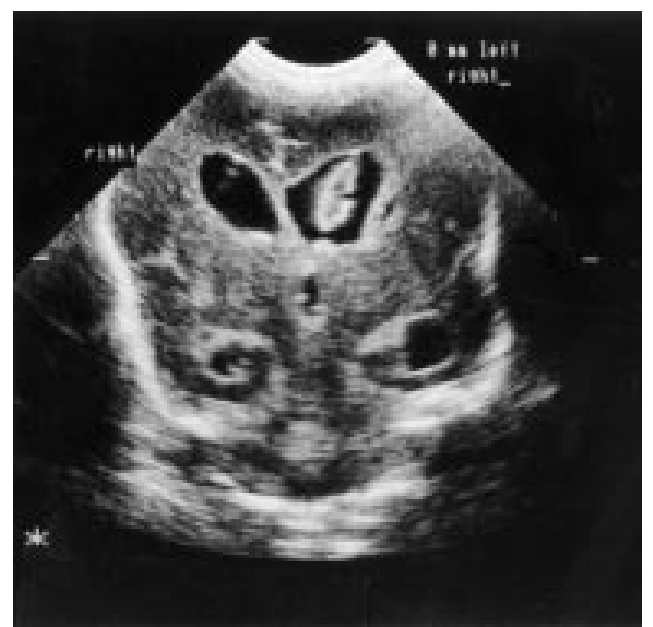

Figure 2 Coronal ultrasound scan with grade 3 intraventricular haemorrhage (IVH).

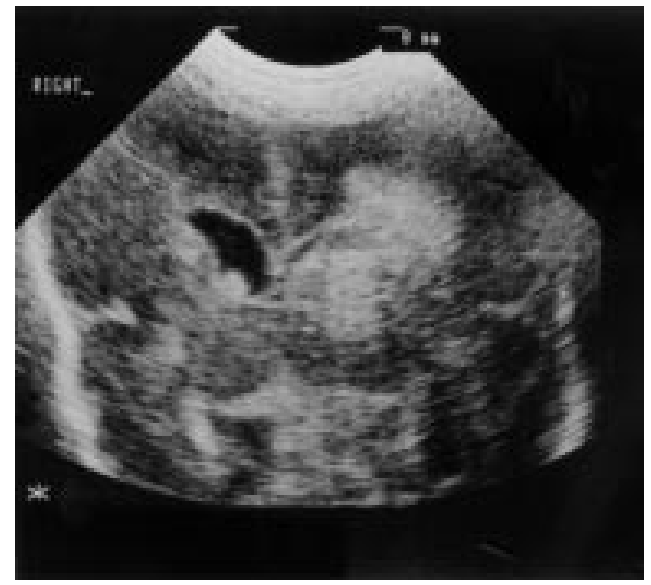

Figure 3 Coronal ultrasound scan with grade 4 intraventricular haemorrhage (IVH).

had stage 3 ROP. IVH was documented in 32 of the 179 neonates -10 without ROP, seven with stage 1 ROP, two with stage 2 ROP, and 13 with stage 3 ROP (Table 1).

IVH was present in $54.2 \%(13 / 24)$ of the infants with stage 3 ROP.

The median gestational age in the group with IVH was 25 (IQR: 25, 26) and those without IVH was 27 (IQR: 25, 27) weeks; this slight difference was not significant $(p=0.06)$. The birth weight in the group with an IVH was slightly lower than those without an IVH, but this did not reach significance, $710 \mathrm{~g}$ (IQR: $650875) \cup 780 \mathrm{~g}$ (IQR: 710850 ), $\mathrm{p}=0.56$ (Fig 4). There were seven female infants. Seven neonates were white, five Asian, and one AfroCaribbean (Table 2).

The severity of IVH in neonates with stage 3 ROP was grade 1 in $61.5 \%(8 / 13)$, grade 2 in $15.4 \%$ (2/13), grade 3 in $7.7 \%$ (1/13), and grade 4 in $15.4 \%(2 / 13)$ (Fig 5$)$.

Twelve out of 13 patients (92.3\%) with stage 3 ROP and IVH were treated for threshold disease, compared with five out of the 11 (45.5\%) patients with stage 3 ROP but without IVH. These data thus provide strong evidence of an association between threshold disease and IVH ( $p=0.02$ ), but little between IVH and sex ( $p$ $=1)$ or ethnic origin $(\mathrm{p}=0.856)$. 
Table 1 Intraventricular haemorrhage (IVH) and retinopathy of prematurity (ROP)

\begin{tabular}{lllll}
\hline & No ROP & Stage 1 & Stage 2 & Stage 3 \\
\hline No of infants & 121 & 22 & 8 & 24 \\
IVH (n) & $31.2 \%(10)$ & $21.8 \%(7)$ & $6.2 \%(2)$ & $40.6 \%(13)$ \\
\hline
\end{tabular}

$\mathrm{n}=$ number of patients.

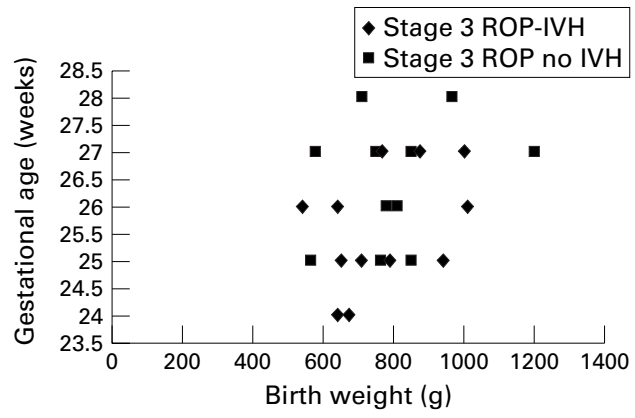

Figure 4 Graph comparing the gestational age with birth weight of those stage 3 ROP infants with and without intraventricular haemorrhage (IVH).

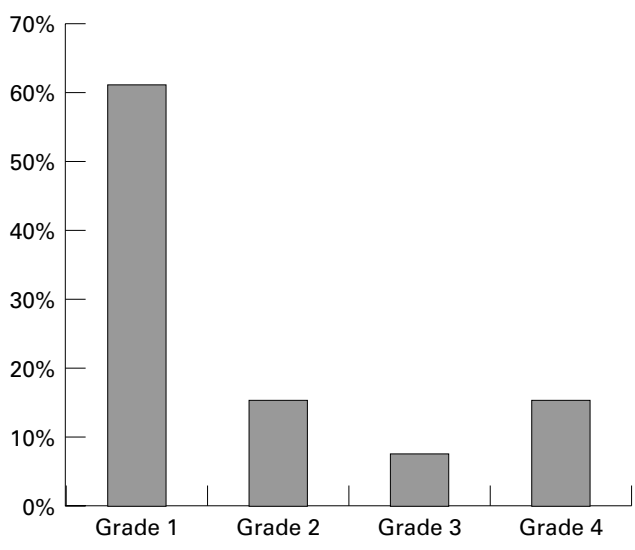

Figure 5 Frequency of various grades of intraventricular haemorrhage (IVH) in stage $3 \mathrm{ROP}$.

\section{Discussion}

The incidence of IVH in very low birthweight infants has been reported to vary between 30\% and $43 \%{ }^{4}{ }^{910}$ The mechanism of IVH has been postulated to be due to the development of an ischaemic environment associated with cerebral hypoperfusion which may lead to rupture of the immature thin walled subependymal vessels causing an IVH. ${ }^{511}$ It is the IVH associated with parenchymal involvement (grade 4) that is believed to be associated with a significant risk of neurodevelopmental handicap. ${ }^{12} 13$ Periventricular leucomalacia

Table 2 Stage 3 retinopathy of prematurity with intraventricular haemorrhage (IVH)

\begin{tabular}{lllllll}
\hline S No & $\begin{array}{l}\text { IVH } \\
\text { grades }\end{array}$ & Treatment & Sex & Ethnic origin & $\begin{array}{l}\text { Gestational } \\
\text { age (weeks) }\end{array}$ & Birth weight (g) \\
\hline 1 & 4 & Laser & M & A & 25 & 710 \\
2 & 2 & Cryotherapy & M & W & 26 & 1010 \\
3 & 1 & Cryotherapy & F & W & 24 & 670 \\
4 & 1 & Cryotherapy & F & A & 26 & 640 \\
5 & 4 & No treatment & M & W & 25 & 790 \\
6 & 1 & Cryotherapy & F & W & 26 & 540 \\
7 & 1 & Cryptherapy & F & A & 25 & 705 \\
8 & 3 & Cryotherapy & M & A & 25 & 940 \\
9 & 1 & Laser & M & W & 27 & 765 \\
10 & 1 & Cryotherapy & F & AC & 25 & 650 \\
11 & 1 & Cryotherapy & F & W & 24 & 640 \\
12 & 1 & Laser & F & A & 27 & 1000 \\
13 & 2 & Cryotherapy & M & W & 27 & 875 \\
\hline
\end{tabular}

$\mathrm{A}=$ Asian, $\mathrm{W}=$ white, $\mathrm{AC}=$ Afro-Caribbean.
(PVL), a hallmark of perinatal ischaemia, is a necrosis of the white matter of the brain which is reported to occur in about $6 \%$ of VLBW infants. ${ }^{1}$

The incidence of any stage of ROP was $65.8 \%$ and of threshold disease was $6 \%$ in the multicentre trial of cryotherapy for ROP in VLBW infants. ${ }^{14}$ The pathogenesis in ROP is believed in part to be due to the hypoxic environment induced by the primary avascular retina. ${ }^{15}$ It has been suggested that there is a linear relation between stage 3 ROP and grade $4 \mathrm{IVH}$, caused to an extent by a hypoxic environment. $\mathrm{Ng}$ et $a l^{5}$ suggested that both severe acute ROP and PVL were related to ischaemia. They reported six cases of stage 3 or greater ROP with PVL and IVH with periventricular echodensities (IVH grade IV), and suggested that additional ischaemia in the carotid circulation contributed to the severity of the eye disease. In addition to ROP, strabismus is also associated with IVH. Phillips et al reported a high prevalence of esotropia with grade 3 and 4 IVH, but lower grade haemorrhages were not a predictor of strabismus suggesting that the degree of parenchymal damage and ocular morbidity may be related. ${ }^{16}$ Hungerford et $a l^{4}$ reported $78 \%$ (seven of nine infants with ROP) of infants with severe ROP had periventricular haemorrhages. A similar association between ROP and periventricular haemorrhage has been reported by Procianoy et al. ${ }^{9}$ There are no reports on the presence of other grades of IVH in stage 3 ROP.

In our study, 13 patients with stage 3 ROP had IVH, and although there was a positive association with IVH and threshold disease ( $\mathrm{p}$ $=0.02)$ the majority $(61.5 \%)$ had grade 1 intraventricular haemorrhage. This heralds a more favourable neurological outcome for the infants as grade 1 and 2 resolve spontaneously and do not lead to any long term sequelae. Two patients had PVL, one with threshold, and the other with subthreshold ROP, one infant had grade 3 IVH with threshold ROP. It is these infants who are at risk of strabismus and neurological deficits. These three infants with severe IVH (grade 3 and above) were all males, two were Asian and one white. They were born at 25 weeks' gestation and had birth weights of 710,790 , and $840 \mathrm{~g}$. There was no difference in the gestational age (GA) or birth weight (BW) between these infants and the other infants with either grade 1 or grade 2 IVH ( $p=$ 0.3 and 0.31 respectively). There was no difference in the prevalence of hyaline membrane disease, chronic lung disease, or sepsis in these infants. It is not known why these three infants developed severe IVH, and perhaps it could be hypothesised that their risk factors were determined prenatally as suggested by Paneth et al. ${ }^{11}$

Our study failed to confirm the previously reported association between severe ROP (stage 3 or above) and severe IVH. This difference between our study and others reporting more severe forms of IVH and advanced acute ROP could be due to a number of factors which include the different population group studied and may include improvement in neo- 
natal and obstetric management. ${ }^{17}$ Recent research on the prevention of IVH indicates that neurological disability may have a predominantly prenatal aetiology and may not be predominantly perinatal as was previously thought. ${ }^{11}$

Recently, Cooke ${ }^{6}$ reported the improved survival of VLBW infants to be accompanied by a decreased incidence of parenchymal haemorrhages and subsequent cerebral palsy rates in survivors. This was significantly associated with the widespread use of antenatal steroids. In our hospital it is the policy that all the mothers in preterm labour receive antenatal steroids if time allows, this might account for the above trend. The administration of antenatal steroids has also been reported to be associated with a decrease in the severity of ROP. ${ }^{18}$

The lower percentage of severe IVH in our study was not mirrored by a decrease in the percentage of stage 3 threshold ROP (11\%). This may perhaps suggest that the earlier studies $^{45}$ which linked severe ROP and IVH, may not presently apply, with improved standards of neonatal care. It also suggests that the hypothesis that both severe ROP and IVH were due to similar hypoxic events may also not be tenable.

The improving standards of neonatal care associated with the increased survival of VLBW infants have led to a lower prevalence of IVH and neurodevelopmental disorders. This change in perinatal outcome appears to have changed the previously well recognised association between IVH and ROP in our cohort of infants with stage 3 ROP.

\section{Conclusions}

In our study although the number of patients with IVH is too small to draw any definite conclusions, those infants with IVH were smaller and more immature (birth weight and gestational age) than those without IVH. All grades of IVH, but predominantly the early grades, which signify a more favourable neurological outcome, may complicate stage 3 ROP. We found a significant association between the presence of IVH and treatment of threshold ROP. We did not, however, confirm the previously documented association between the severity of IVH and severity of ROP. However, the presence of even a minor grade of IVH may be a significant risk factor for threshold ROP once stage 3 disease has developed.

1 Stevenson DK, Wright LL, Lemons JA, et al. Very low birth weight outcomes of the national institute of child health and human development neonatal research network January 1993 through December 1994. Am 7 Obstet Gynecol 1998;179(part 1):1632-9.

2 1998;19(part 1):1632-9. of New Zealand infants with birth weight less than $1500 \mathrm{~g}$ screened for retinopathy of prematurity: visual outcome at screened for retinopathy of prematurity: visual o

3 Papile L, Burstein J, Burstein R, et al. Incidence and evolution of subependymal and intraventicular haemorrhage: A study of infants with weights less than $1,500 \mathrm{gm}$. F Pediatrics 1978;92:529-34.

4 Hungerford J, Stewart A, Hope P. Ocular sequelae of preterm birth and their relation to ultrasound evidence of cerebral damage. Br $\mathcal{F}$ Ophthalmol 1986;70:463-8.

$5 \mathrm{Ng}$ YK, Fielder AR, Levene MI, et al. Are severe acute retinopathy of prematurity and severe periventricular leucomalacia both ischaemic insults. Br 7 Ophthalmol 1989;73:11114.

6 Cooke RWI. Trends in the incidence of cranial ultrasound lesions and cerebral palsy in very low birthweight infants 1982-93. Arch Dis Child Fetal Neonatal Ed 1999;80:F11517.

7 Committee for the Classification of Retinopathy of Prematurity. The international classification of retinopathy of prematurity. Arch Ophthalmol 1984;102:1130-4.

8 Royal College of Ophthalmologists and British Association of Perinatal Medicine. Retinopathy of prematurity guidelines for screening and treatment. The report of the joint working party. London: RCO, 1995.

9 Procianoy RS, Garcia-Prats JA, Hittner HM, et al. An association between retinopathy of prematurity and intraventricular haemorrhage in very low birth weight infants. Acta Paediatr Scand 1981;70:473-7.

10 Hutchison AA, Barrett JM, Fleischer AC. Intraventricular hemorrhage in the premature infant. $N$ Engl $f \mathrm{Med}$ 1982;307:1272-3.

11 Paneth N, Pinto-Martin J, Gardiner J, et al. Incidence and timing of germinal matrix/Intraventricular hemorrhage in low birth weight infants. Am $\mathcal{F}$ Epidemiol 1993;137:1167 76

12 Krishnamoorthy KS, Shannon DC, Delong GR, et al. Neurologic sequelae in the survivors of neonatal intraventricurologic sequelae in the survivors of neona

13 Szymonowicz W, Yu VYH, Bajuk B, et al. Neurodevelopmental outcome of periventricular haemorrhage leukomalacia in infants $1250 \mathrm{~g}$ or less at birth. Early Hum Dev lacia in infants

14 Palmer EA, Flynn JT, Hardy RJ, et al. The cryotherapy for retinopathy of prematurity cooperative group. Incidence and early course of retinopathy of prematurity. Ophthalmology 1991;98:1628-40.

15 Foos RY. Retinopathy of prematurity. Pathological correlation of clinical stages. Retina 1987;7:260-76.

16 Phillips J, Christiansen SP, Ware G, et al. Ocular morbidity in very low birth weight infants with intraventricular hemorrhage. Am f Ophthalmol 1997;123:218-23.

17 Shaver DC, Bada HS, Korones SB, et al. Early and late intraventricular hemorrhage: the role of obstetric factors. Obstet Gynecol 1992;80:831-7.

18 Higgins RD, Mendelsohn AL, DeFeo MJ, et al. Antenatal dexamethasone and decreased severity of retinopathy. Arch Ophthalmol 1998;116:601-5. 